\title{
Remerciements à nos évaluateurs de 2018
}

Nous tenons à remercier les personnes suivantes pour leur contribution inestimable en tant qu'évaluateurs pour la revue Promotion de la santé et prévention des maladies chroniques au Canada en 2018. Leur expertise contribue grandement à la qualité de notre revue et à la diffusion des nouvelles connaissances auprès de la communauté scientifique, au Canada comme à l'échelle internationale.

\begin{tabular}{|c|c|c|}
\hline Ashley Aimone & Tara Gomes & Catherine Pelletier \\
\hline Philippe Autier & Andrea Gruneir & Paul Peters \\
\hline Juanita Bacsu & Heather Hannah & Sarah A. Richmond \\
\hline Farin Bakhtiari & Lisa D. Hawke & Nancy A. Ross \\
\hline Maulik Baxi & Katelyn Holliday & Tayyab Shah \\
\hline Patrick Berrigan & Birgit Isernhagen & Michèle Shemilt \\
\hline Jennifer Bethell & Shanthi Johnson & Kelley J. Sittner \\
\hline Monique Bordeleau & Tarun Katapally & Amanda K. Slaunwhite \\
\hline Songul Bozat-Emre & Scott Kehler & Richard S. Stanwick \\
\hline Ruben Brondeel & Soyeon Kim & Mark C.J. Stoddart \\
\hline Lauren Burt & Melissa Kimber & Carol Strike \\
\hline Yue Chen & Rachel Laxer & Mariano Suppa \\
\hline Phil Chilibeck & Eun-Young Lee & Samuel Tomczyk \\
\hline Susan Clayton & Shelly-Anne Li & Linda Varangu \\
\hline Erica Di Ruggiero & Alyson Mahar & Paul Villeneuve \\
\hline Carolyn Dohoo & John McLaughlin & Daniel Warshafsky \\
\hline Frank J. Elgar & Jacqueline Middleton & Rania A. Wasfi \\
\hline Tara Elton-Marshall & Elaine Moody & Colleen Whyte \\
\hline Philippe Finès & Patrick Morency & Michel D. Wissing \\
\hline Jean-Michel Galarneau & Howard Morrison & Niko Yiannakoulias \\
\hline Fidji Gendron & Karen A. Patte & Jennifer Zwicker \\
\hline
\end{tabular}

